\title{
Gestational diabetes mellitus may be associated with increased risk of breast cancer
}

\author{
Yong-Moon Mark Park ${ }^{1}$, Katie M O'Brien ${ }^{2}$, Shanshan $Z_{\text {hao }}{ }^{2}$, Clarice R Weinberg $^{2}$, Donna D Baird ${ }^{1}$ \\ and Dale P Sandler ${ }^{\star}, 1$ \\ ${ }^{1}$ Epidemiology Branch, Division of Intramural Research, National Institute of Environmental Health Sciences, National Institutes \\ of Health, Research Triangle Park, NC 27709, USA and 'Biostatistics \& Computational Biology Branch, Division of \\ Intramural Research, National Institute of Environmental Health Sciences, National Institutes of Health, Research Triangle Park, \\ NC 27709, USA
}

Background: Although a positive association between type 2 diabetes and breast cancer has been reported, an association with gestational diabetes mellitus (GDM) is less clear.

Methods: The Sister Study enroled 50884 women aged 35-74 years, from 2003 through 2009. Cox proportional hazards models were used to estimate breast cancer risk in relation to GDM.

Results: Ever having GDM was not associated with breast cancer overall (hazards ratio $(H R)=1.10,95 \%$ confidence interval $(C I)=0.88-1.36)$, but there was a suggestive association between ever having a GDM pregnancy and oestrogen receptor (ER) negative breast cancer $(\mathrm{HR}=1.73,95 \% \mathrm{Cl}=0.98-3.06)$. However, having 2 or more $\mathrm{GDM}$ pregnancies was associated with overall breast cancer risk $1.68(95 \% \mathrm{Cl}=1.15-2.44)$ and with ER-positive breast cancer $(\mathrm{HR}=1.81,95 \% \mathrm{Cl}=1.10-2.98)$, which was supported by sensitivity analyses. Results were similar when analyses were stratified by whether or not type 2 diabetes had developed after GDM.

Conclusions: Women with multiple GDM pregnancies had a higher incidence of breast cancer, suggesting that such women could benefit from increased surveillance for breast cancer.

Gestational diabetes mellitus (GDM) is associated with adverse pregnancy outcomes (Agha-Jaffar et al, 2016) and long-term adverse health conditions, including type 2 diabetes (Bellamy et al, 2009), metabolic syndrome (Xu et al, 2014), cardiovascular diseases (Li et al, 2014) and some cancers (Tong et al, 2014). GDM is characterised by insulin resistance and glucose intolerance that may persist after delivery. Chronic health outcomes associated with GDM could be due to persistent beta-cell dysfunction and impaired insulin sensitivity with onset during the reproductive years (Catalano, 2010). Although a positive association between type 2 diabetes and breast cancer has been reported (Hardefeldt et al, 2012), an association with GDM is less clear (Tong et al, 2014). In addition, since recurrent GDM is a potent predictor of type 2 diabetes, perhaps due to exposure to multiple episodes of insulin resistance (Bottalico, 2007), we hypothesised that multiple GDM pregnancies would be associated with increased risk of breast cancer. The objective of this study was to investigate the association between GDM and breast cancer risk.

\section{MATERIALS AND METHODS}

The Sister Study is a nationwide prospective cohort study investigating environmental and genetic risk factors for breast cancer (Weinberg et al, 2007). Enrolment targeting 35-74-year olds during 2003-2009 accrued 50884 breast-cancer-free sisters of

*Correspondence: Dr DP Sandler; E-mail: sandler@niehs.nih.gov

Received 27 October 2016; revised 23 December 2016; accepted 15 January 2017; published online 16 February 2017

(C) 2017 Cancer Research UK. All rights reserved 0007-0920/17 
women diagnosed with breast cancer. Incident breast cancer cases included in this analysis were ascertained until 14 August 2015 (Data release 5.0). Characteristics of participants, including history of GDM and diabetes mellitus, were obtained using telephone interviews completed at baseline. Incident breast cancers were subsequently reported on annual health updates (response rates $>92 \%$ ). Self-reported incident breast cancers were verified by medical records when available ( $81 \%$ of cases at the time of this data release). Agreement between self-reports and medical records was high $(99.5 \%)$, so self-reported diagnoses were included when records were not obtained. Participants were questioned about each pregnancy to ascertain whether they had had pregnancyrelated diabetes or an abnormal glucose tolerance test during the pregnancy. Participants with diabetes prior to their pregnancy were not considered GDM cases and were excluded from the analysis $(n=187)$. For the present analysis, we included parous women $(n=41640)$ and excluded those who had incomplete information for GDM or diabetes, or who had reported a history of any cancer except non-melanoma skin cancer at baseline $(n=2255)$. The remaining 39198 women contributed 291150 person-years of follow-up.

We used multivariable Cox proportional hazards models to assess hazard ratios (HRs) and 95\% confidence intervals (CIs) for the associations between GDM and invasive breast cancer, with adjustment for race/ethnicity, 10-year birth cohorts, educational attainment, age at first birth, age at menarche, relative weight at age $10, \mathrm{BMI}$ at 30-39 years old and physical activity (metabolic equivalent hours/week) in their childhood and teens. Age was used as the timescale. Women with in situ breast cancer were censored at the time of diagnosis in analyses of invasive breast cancer. Potential effect modification by menopause status (pre- and postmenopause) was evaluated by adjusting for menopause as a time-varying covariate; likelihood ratio tests were used to test for effect modification. In addition, potential effect modification by birth cohort due to changes in diagnostic criteria and/or changes in treatments for GDM over time (Mestman, 2002) was evaluated by testing for interaction between GDM and a categorical variable based on 10-year birth cohorts, using a likelihood ratio test. Case-only analysis (Martinez et al, 2010) was applied to evaluate whether the association between GDM and breast cancer differed according to oestrogen receptor (ER) expression. To explore whether type 2 diabetes might mediate the association between GDM and risk of breast cancer, we stratified the Cox model by type 2 diabetes status at baseline, allowing the baseline hazard during follow-up to be different for those who reported that they had or had not developed type 2 diabetes between their first GDM pregnancy and baseline. Statistical significance was evaluated with two-sided tests, with $\alpha=0.05$. SAS 9.3 software (SAS Institute Inc., Cary, NC, USA) was used throughout.

\section{RESULTS}

Among the study subjects, $4.2 \%$ had at least one pregnancy with GDM and $0.9 \%$ had multiple GDM pregnancies. Women with two or more pregnancies with GDM were more likely to report being heavier than their peers in childhood, to have been obese at 30-39 years old and at baseline, to have had pregnancy complications, and to have subsequently been diagnosed with diabetes mellitus (Table 1).

A total of 2141 women developed incident breast cancer during a mean follow-up of 7.4 years (1609 invasive and 532 in situ cancers). While ever having a pregnancy with GDM was not associated with breast cancer risk overall, GDM was marginally associated with an increased risk of ER-negative invasive breast cancer (covariateadjusted $\mathrm{HR}=1.73$, 95\% CI: 0.98-3.06). Compared with women without GDM, women who had experienced multiple GDM
Table 1. Characteristics of the Sister Study participants at baseline according to self-reported GDM status

\begin{tabular}{|c|c|c|c|}
\hline \multirow{2}{*}{$\begin{array}{l}\text { Characteristic } \\
\text { No. of GDM }\end{array}$} & \multirow{2}{*}{$\begin{array}{c}\text { Without } \\
\text { GDM } \\
0\end{array}$} & \multicolumn{2}{|c|}{ With GDM } \\
\hline & & 1 & $\geqslant 2$ \\
\hline No. of participants, $n \%$ & $37562(95.8)$ & $1283(3.3)$ & $353(0.9)$ \\
\hline \multicolumn{4}{|l|}{ Mean (s.d.) } \\
\hline Age at baseline, year & $56.1(9.0)$ & $51.6(8.2)$ & $51.2(7.9)$ \\
\hline $\mathrm{BMI}$ at baseline, $\mathrm{kg} \mathrm{m}^{-2}$ & $27.8(6.0)$ & $29.3(7.3)$ & $29.7(6.8)$ \\
\hline $\mathrm{BMI}$ at $30-39$ years old, $\mathrm{kg} \mathrm{m}^{-2}$ & $23.2(3.8)$ & $24.6(5.1)$ & $25.0(5.1)$ \\
\hline Waist circumference at baseline, $\mathrm{cm}$ & $86.4(14.4)$ & $89.7(16.4)$ & $91.3(15.7)$ \\
\hline Age at menarche, year & $12.7(1.5)$ & $12.6(1.5)$ & $12.6(1.7)$ \\
\hline Age at first birth, year & $24.6(5.2)$ & $26.4(5.9)$ & $25.8(5.3)$ \\
\hline Parity & $2.4(1.1)$ & $2.5(1.2)$ & $2.8(1.1)$ \\
\hline $\begin{array}{l}\text { Total MET-hours of physical activity, } \\
\text { week at baseline }\end{array}$ & $51.2(31.2)$ & $48.3(31.4)$ & $50.9(30.5)$ \\
\hline $\begin{array}{l}\text { Total MET-hours of physical activity, } \\
\text { week at childhood and teens }\end{array}$ & $8.4(15.5)$ & $9.4(16.7)$ & $8.3(13.9)$ \\
\hline \multicolumn{4}{|l|}{ Proportion (\%) } \\
\hline Non-Hispanic white & 83.6 & 77.0 & 72.5 \\
\hline Having $\geqslant$ college degree & 47.4 & 49.4 & 49.6 \\
\hline Relatively heavier at age 10 & 17.3 & 18.6 & 20.2 \\
\hline Postmenopausal at enrolment & 68.2 & 47.7 & 49.0 \\
\hline $\begin{array}{l}\text { Having } \geqslant 2 \text { first-degree family } \\
\text { members with breast cancer }\end{array}$ & 27.0 & 24.4 & 23.5 \\
\hline Having a mammogram within a year & 81.2 & 78.3 & 76.5 \\
\hline $\begin{array}{l}\text { Giving birth to a baby weighing more } \\
\text { than } 4 \mathrm{~kg}\end{array}$ & 15.6 & 24.7 & 29.1 \\
\hline History of gestational hypertension & 7.1 & 18.6 & 20.4 \\
\hline History of pre-eclampsia or eclampsia & 8.1 & 15.1 & 17.6 \\
\hline History of parental diabetes & 34.6 & 47.7 & 52.7 \\
\hline Self-reported diabetes & 5.6 & 16.5 & 22.3 \\
\hline
\end{tabular}

Abbreviations: $\mathrm{BMI}=$ body mass index; $\mathrm{GDM}=$ gestational diabetes mellitus; $\mathrm{MET}=$ metabolic equivalent. Data are presented as mean (s.d.), or percentage (\% within strata of GDM, except for total number of participants).

pregnancies had increased risk of total and invasive breast cancer $(\mathrm{HR}=1.68,95 \% \mathrm{CI}: 1.15-2.44 ; \mathrm{HR}=1.72,95 \% \mathrm{CI}: 1.11-2.65$, respectively; Table 2). This association was seen in both premenopausal and postmenopausal breast cancer (Supplementary Table 1). Multiple episodes of GDM were also associated with ER-positive breast cancer $(\mathrm{HR}=1.81,95 \% \mathrm{CI}$ : 1.10-2.98). An association between GDM and ER-negative breast cancer was apparent among women with a single GDM pregnancy $(\mathrm{HR}=1.88$, 95\% CI: $1.02-$ 3.47), but the HR for ER-negative breast cancer and multiple pregnancies could not be estimated due to small numbers (Table 2). When we restricted analysis to women who had two full-term pregnancies, to address potential bias due to selective fertility (for example, if women with a GDM-related pregnancy complication chose not to have a subsequent pregnancy) and differential opportunity for exposure, the estimated associations between GDM and breast cancer were more pronounced (Supplementary Table 2). When we included women who reported borderline GDM, which may represent a history of subclinical glucose intolerance, the association between multiple GDM pregnancies and risk of breast cancer persisted, although the effect estimates were attenuated (data not shown). There was no evident effect modification by timevarying menopause status and no evidence for interaction between GDM and birth cohort.

When we stratified the Cox regression model on type 2 diabetes at baseline, the results were not materially changed (Supplementary Table 3). When we excluded women who reported subsequent type 2 diabetes, the overall results were not materially different from the main analysis (data not shown). Furthermore, inclusion of body mass index and waist circumference at baseline (no one was pregnant at baseline, by design) in the multivariable models also did not materially change the results (data not shown). 
Table 2. HRs and $95 \% \mathrm{Cls}$ for the association between GDM and breast cancer

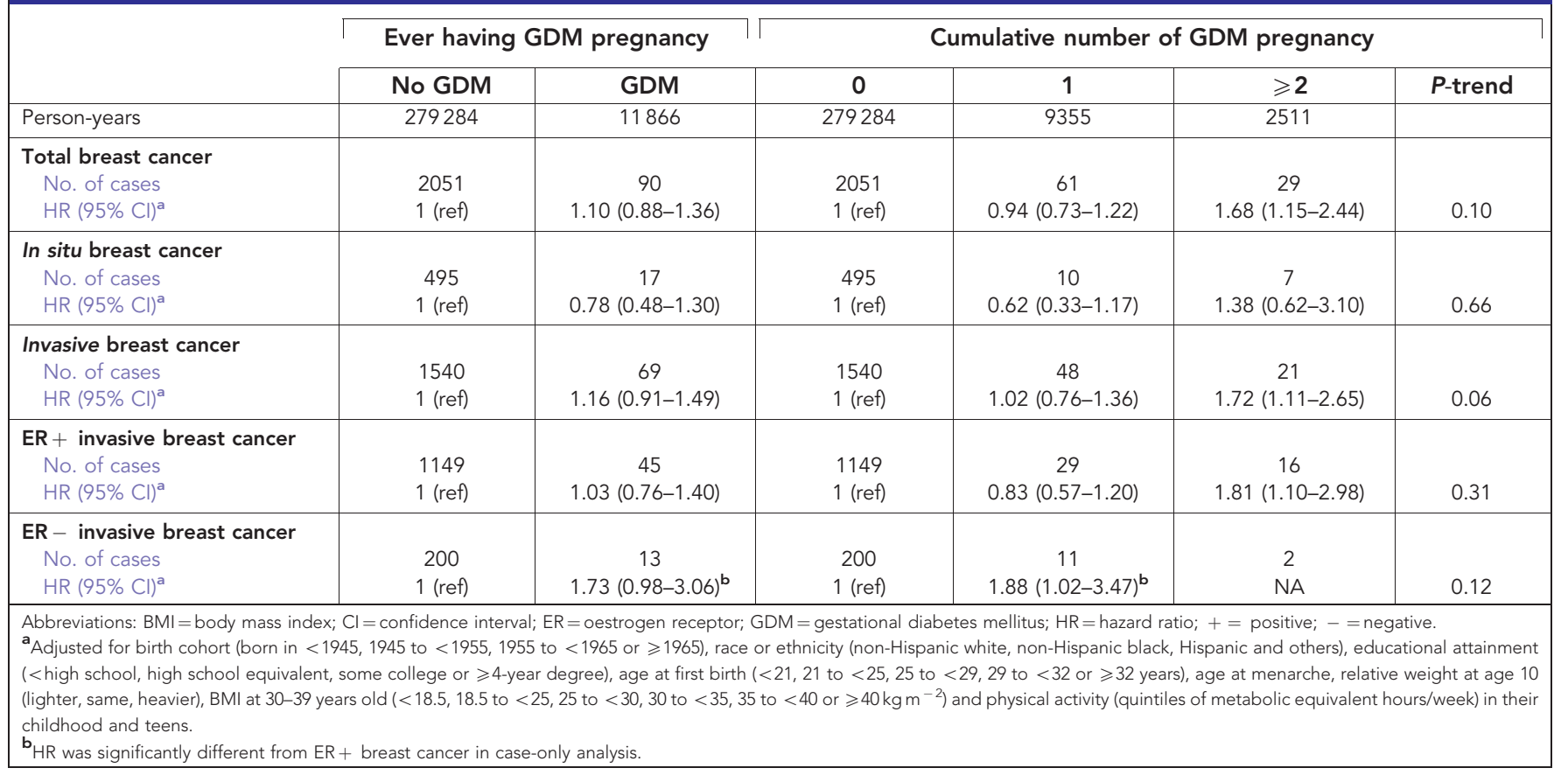

\section{DISCUSSION}

To our knowledge, this is the first report of an association between multiple GDM pregnancies and increased risk of breast cancer. There was also an association between GDM and ER-negative breast cancer, but there were too few cases to evaluate risk related to the number of affected pregnancies.

Our findings have biological plausibility. Women with multiple GDM pregnancies have exhibited impaired glucose tolerance during pregnancy, and will tend to have had higher BMI, and greater weight gain during and between pregnancies (Schwartz et al, 2016) which might make them more vulnerable to chronic hyperinsulinemia and insulin resistance. Repeated episodes of GDM likely amplify these effects or serve as a marker for subclinical abnormalities in glucose metabolism (Bottalico, 2007). Resulting increases in bioactivity of insulin growth factors may have mitogenic and anti-apoptotic effects that could influence the remodelling of breast tissue late in pregnancy and contribute to the initiation and progression of breast cancer (Wolf et al, 2005; Ryu et al, 2014).

A previous meta-analysis (Tong et al, 2014) reported no association between ever having GDM and breast cancer, while inverse associations were reported for premenopausal women in two studies (Rollison et al, 2008; Bejaimal et al, 2016) and for both pre- and postmenopausal women in another study (Powe et al, 2016). However, the prior studies had limited ability to examine breast cancer subtypes, control for confounding (Bejaimal et al, 2016) or enrol appropriate controls (Rollison et al, 2008); none evaluated the role of multiple GDM pregnancies. Furthermore, our analysis that was restricted to women with exactly two full-term births should have served to minimise parity-based differential opportunity to develop GDM. In the present study, adjusting for subsequent type 2 diabetes did not change the risk estimates for the association between GDM and risk of breast cancer, and the association remained in analysis restricted to women who did not report subsequent clinical diabetes. However, our ability to address the possible mediation of this association through later onset diabetes was limited. We relied on self-report of diabetes rather than laboratory measurements of fasting blood glucose, so some women with diabetes will be misclassified. In addition, estimating an effect of clinical diabetes on breast cancer risk is challenging due to the common use of anti-diabetic medications that may have anti-neoplastic properties (Gonzalez-Angulo and Meric-Bernstam, 2010). GDM is likely a marker for women at risk for changes in glucose metabolism that could have implications for long-term disease risk. It might also be a marker for unknown risk factors for breast cancer that could not be accounted for in the present study.

A potential limitation is that history of GDM was based on selfreport. However, agreement between self-report and medical records was $94 \%$ in another study (Powe et al, 2016). Strengths of the present study include the prospective design with high retention, large sample size, standardized data collection and comprehensive information on potential risk factors for breast cancer.

In summary, among parous women, a history of multiple episodes of GDM was associated with increased risk of breast cancer, suggesting that abnormal glucose metabolism might be aetiologically important for breast cancer and that history of multiple GDM pregnancies might be a marker for identifying women who are at increased risk of breast cancer and therefore should be screened more frequently.

\section{ACKNOWLEDGEMENTS}

This research was supported by the Intramural Research Program of the NIH, National Institute of Environmental Health Sciences (Z01-ES044005 and -ES102245).

\section{CONFLICT OF INTEREST}

The authors declare no conflict of interest. 


\section{AUTHOR CONTRIBUTIONS}

Y-MMP and DPS contributed to the study concept and design. Y-MMP contributed to statistical analysis. Y-MMP drafted the manuscript. All authors contributed to interpretation of the data and critically revised the manuscript for important intellectual content. All authors approved the final version of the manuscript. DPS is the guarantor of this work and, as such, had full access to all the data in the study and takes responsibility for the integrity of the data and the accuracy of the data analysis. We are grateful to Drs Allen J Wilcox and Quaker E Harmon for critical review of this manuscript.

\section{REFERENCES}

Agha-Jaffar R, Oliver N, Johnston D, Robinson S (2016) Gestational diabetes mellitus: does an effective prevention strategy exist? Nat. Rev. Endocrinol. 12(9): 533-546.

Bejaimal SA, Wu CF, Lowe J, Feig DS, Shah BR, Lipscombe LL (2016) Shortterm risk of cancer among women with previous gestational diabetes: a population-based study. Diabet Med 33(1): 39-46.

Bellamy L, Casas JP, Hingorani AD, Williams D (2009) Type 2 diabetes mellitus after gestational diabetes: a systematic review and meta-analysis. Lancet 373(9677): 1773-1779.

Bottalico JN (2007) Recurrent gestational diabetes: risk factors, diagnosis, management, and implications. Semin Perinatol 31(3): 176-184.

Catalano PM (2010) Obesity, insulin resistance, and pregnancy outcome. Reproduction 140(3): 365-371.

Gonzalez-Angulo AM, Meric-Bernstam F (2010) Metformin: a therapeutic opportunity in breast cancer. Clin Cancer Res 16(6): 1695-1700.

Hardefeldt PJ, Edirimanne S, Eslick GD (2012) Diabetes increases the risk of breast cancer: a meta-analysis. Endocr Relat Cancer 19(6): 793-803.

Li JW, He SY, Liu P, Luo L, Zhao L, Xiao YB (2014) Association of gestational diabetes mellitus (GDM) with subclinical atherosclerosis: a systemic review and meta-analysis. BMC Cardiovasc Disord 14: 132.
Martinez ME, Cruz GI, Brewster AM, Bondy ML, Thompson PA (2010) What can we learn about disease etiology from case-case analyses? Lessons from breast cancer. Cancer Epidemiol Biomarker Prev 19(11): 2710-2714.

Mestman JH (2002) Historical notes on diabetes and pregnancy. Endocrinologist 12(3): 224-242.

Powe C, Tobias DK, Michels K, Chen WY, Eliassen AH, Manson JE, Rosner B, Willett WC, Hu FB, Zhang C, Rich-Edwards JW, Rexrode KM. History of gestational diabetes mellitus (GDM) and risk of incident invasive breast cancer among parous women in the Nurses' Health Study II prospective cohort. Cancer Epidemiol Biomarkers Prev; e-pub ahead of print 11 October 2016; doi:10.1158/1055-9965.EPI-16-0601.

Rollison DE, Giuliano AR, Sellers TA, Laronga C, Sweeney C, Risendal B, Baumgartner KB, Byers T, Slattery ML (2008) Population-based casecontrol study of diabetes and breast cancer risk in Hispanic and non-Hispanic White women living in US southwestern states. Am J Epidemiol 167(4): 447-456.

Ryu TY, Park J, Scherer PE (2014) Hyperglycemia as a risk factor for cancer progression. Diabetes Metab J 38(5): 330-336.

Schwartz N, Nachum Z, Green MS (2016) Risk factors of gestational diabetes mellitus recurrence: a meta-analysis. Endocrine 53(3): 662-671.

Tong GX, Cheng J, Chai J, Geng QQ, Chen PL, Shen XR, Liang H, Wang DB (2014) Association between gestational diabetes mellitus and subsequent risk of cancer: a systematic review of epidemiological studies. Asian Pac J Cancer Prev 15(10): 4265-4269.

Weinberg CR, Shore DL, Umbach DM, Sandler DP (2007) Using risk-based sampling to enrich cohorts for endpoints, genes, and exposures. Am J Epidemiol 166(4): 447-455.

Wolf I, Sadetzki S, Catane R, Karasik A, Kaufman B (2005) Diabetes mellitus and breast cancer. Lancet Oncol 6(2): 103-111.

Xu Y, Shen S, Sun L, Yang H, Jin B, Cao X (2014) Metabolic syndrome risk after gestational diabetes: a systematic review and meta-analysis. PLoS One 9(1): e87863.

This work is published under the standard license to publish agreement. After 12 months the work will become freely available and the license terms will switch to a Creative Commons AttributionNonCommercial-Share Alike 4.0 Unported License.

Supplementary Information accompanies this paper on British Journal of Cancer website (http://www.nature.com/bjc) 